\title{
FK506-loaded PLGA nanoparticles improve long-term survival of a vascularized composite allograft in a murine model
}

\author{
Zheming Cao", Cheng Li", Jiqiang He, Xinlei Sui, Panfeng Wu, Ding Pan, Liming Qing, Juyu Tang \\ Department of Orthopedics, Xiangya Hospital of Central South University, Changsha, China \\ Contributions: (I) Conception and design: J Tang; (II) Administrative support: L Qing, D Pan, P Wu; (III) Provision of study materials or patients: Z \\ Cao, C Li; (IV) Collection and assembly of data: Z Cao, C Li; (V) Data analysis and interpretation: J He, X Sui; (VI) Manuscript writing: All authors; \\ (VII) Final approval of manuscript: All authors. \\ "These authors contributed equally to this work. \\ Correspondence to: Juyu Tang, MD, PhD. Department of Orthopedics, Xiangya Hospital of Central South University, 87\# Xiangya Road, Changsha \\ 410008, China. Email: tangjuyu@csu.edu.cn.
}

\begin{abstract}
Background: The side effects of life-long administration of FK506 limit the clinical practice of vascularized composite allografts (VCAs). This study aimed to evaluate the feasibility of FK506-loaded poly (lacticco-glycolic acid) (PLGA) nanoparticles (FK506 NPs) for prolonging the long-term survival of VCAs and reducing the side effects of FK506.

Methods: PLGA nanoparticles loaded with FK506 were prepared by the solvent evaporation method. The characterization of FK506 NPs was evaluated by electron microscopy. To confirm the function and safety of FK506 NPs, these particles were administrated into rats by intraperitoneal injection. The survival time of the allograft, systemic concentration of FK506, anti-rejection activity, and side-effect of FK506 NPs were evaluated in a Brown Norway (BN)-to-Sprague Dawley (SD) epigastric VCA transplantation model.

Results: Compared with the nontreatment, PLGA control and FK506 groups, the median survival times (MST) of the FK506 NP groups were significantly prolonged. The FK506 NPs could maintain therapeutic drug concentration for 60 days. Moreover, cytokine concentrations, flow cytometry of regulatory $\mathrm{T}$ cells (Tregs) and histopathology of allografts revealed significantly prolonged immunosuppression by FK506 NPs. FK506 NPs also ameliorated FK506 nephrotoxicity.

Conclusions: FK506 NPs prolong the survival time of VCAs in a murine model with minimal nephrotoxicity, and provide a potential clinical strategy for ameliorating long-term side effects of immunosuppressive therapy.
\end{abstract}

Keywords: Flap transplantation; immune tolerance; poly (lactic-co-glycolic acid) nanoparticle (PLGA nanoparticle); FK506; vascularized composite allograft transplantation (VCA transplantation)

Submitted May 12, 2021. Accepted for publication Aug 20, 2021.

doi: 10.21037/atm-21-2425

View this article at: https://dx.doi.org/10.21037/atm-21-2425

\section{Introduction}

Transplantation of vascularized composite allografts (VCAs) is a promising option to restore complex anatomical and functional units with severe injury and defects that cannot be repaired by conventional reconstruction with autologous tissues or prostheses. Over the last two decades, several different VCA transplant, especially hand and face allotransplantations, have been successfully performed with good functional and esthetic outcomes worldwide (1-6). However, due to the allogeneic nature of VCAs, severe immunological rejection occurs after transplant, especially with skin allografts. Over $87.8 \%$ of the upper extremity and $72.7 \%$ of the facial allotransplantation patients experienced at least one episode of acute rejection in the first post-transplant year $(7,8)$. VCA transplant also leads to chronic rejection targeting mainly the allograft 
vasculature, leading to graft vasculopathy and ischemic necrosis of the graft (9). As in solid organ transplantation, life-long immunosuppressive therapy to achieve long-term graft survival in VCAs is indispensable.

FK506 (tacrolimus), the first-line recommendation of calcineurin inhibitors, is commonly used in combination with mycophenolate mofetil and a steroid as a high-dose triple regimen for immunosuppressive maintenance in VCA transplantations, and this has been the mainstay in preventing and treating acute rejection since the 1980s (10-13). However, long-term systemic delivery of tacrolimus results in nephrotoxicity, hyperglycemia, hypertension, infection, and neoplasia (14-18). Additionally, higher rejection risks for VCA limits consideration of alternate immunosuppressive therapies. Therefore, future research in this field may primarily focus on the development of new FK506 formulations to achieve optimal immunosuppressive effects.

Biodegradable polymers, characterized by excellent bioavailability, biosafety, and controlled release, have been commonly used in a variety of nanocarrier drug delivery systems, such as microspheres, microcapsules, nanoparticles, pellets, implants, and films (19,20). Many studies have confirmed the capability of biodegradable polymers to prolong the survival of allografts without significant adverse effects (21-24). Tacrolimus-encapsulated glycolide-co-clatide-co-caprolactone polymer has been confirmed to prolong corneal allograft survival without adverse effects in rabbit eyes (25). In 2014, Xu et al. used degradable methoxy poly (ethylene glycol)—poly (lactide) (MPEG-PLA) to encapsulate FK506 and tested it on livertransplanted Sprague-Dawley (SD) rats. Their results showed that the survival time of the rats treated with FK506-loaded MPEG-PLA nanoparticles was significantly prolonged compared to other groups, with better liver function (26). Encouraging data were also obtained in a Brown Norway-to-Lewis rat hindlimb transplantation model using tacrolimus-hydrogel which prolonged graft survival time to more than 100 days (27).

Poly lactic-co-glycolic acid (PLGA) is a widely used biodegradable material. The release kinetics of PLGAencapsulated drugs are in conformity with the first-order kinetic equation (28). The high tacrolimus trough-level variability has been demonstrated as the detriment for longterm allograft survival in renal transplantations. However, the stable, sustained, and slow release of the encapsulated drugs indicates that PLGA may be a promising delivery system for VCAs (29). Therefore, in the present study, FK506-loaded nanoparticles were encapsulated in PLGA to comprehensively examine the feasibility of using this method to prolong the survival time of allografts.

We present the following article in accordance with the ARRIVE reporting checklist (available at https://dx.doi. org/10.21037/atm-21-2425).

\section{Methods}

Fabrication and characterization of FK506-loaded PLGA nanoparticles

Tacrolimus and PLGA (lactide:glycolide ratio 75:25, mol wt 66,000-107,000) were purchased from Sigma-Aldrich company (St. Louis, MO, USA). FK506 PLGA nanoparticles (FK506 NPs) were prepared by a solvent evaporation process described in detail previously (30-32) by the Pharmaceutical Technology Department of the University of Santiago de Compostela. A lactide/glycolide concentration ratio of 75:25 was used, and related studies confirmed the high encapsulation efficiency (33). The blank PLGA NPs were prepared by the same procedure without tacrolimus loading.

The morphology and size of the samples were observed and recorded using the Hitachi HT7700 electron microscope (Hitachi Ltd., Japan). Units of $6 \mathrm{mg}$ FK506 were encapsulated with PLGA and lyophilized for $48 \mathrm{~h}$, and the lyophilized solid nanoparticles were collected and stored at $-20^{\circ} \mathrm{C}$. UV disinfection was required before administration.

The encapsulation and loading of tacrolimus into FK506 NPs were determined by analyzing the acetonitrile solution dissolved with weighted amount of FK506 NPs. Briefly, the solvents were diluted with acetonitrile, and then injected into a high-performance liquid chromatography (HPLC) with $\mathrm{C}_{18}$ column $(4.6 \mathrm{~mm} \times 250 \mathrm{~mm}, 5 \mu \mathrm{m}$, Kromasil, Sweden $)$ at $50{ }^{\circ} \mathrm{C}$. The mobile phase was a mixture of double distilled water/acetonitrile $=70: 30$ (volume ratio) and the $\mathrm{pH}$ of water was adjusted to 4.0 by orthophosphoric acid, and the flow rate of the mobile phase was $0.7 \mathrm{~mL} /$ minute. Tacrolimus was detected at a wavelength of $210 \mathrm{~nm}$. The drug-loading (\%DL) and encapsulation (\%EE) was calculated with the following equations: \% $\mathrm{DL}=$ (weight of tacrolimus in FK506 $\mathrm{NPs} /$ weight of FK506 NPs) $\times 100$ and \%EE $=$ (weight of tacrolimus in FK506 NPs/weight of tacrolimus used for FK506 NPs preparation) $\times 100$.

\section{Establishment of the animal model}

Purified male SD and Brown-Norway (BN) rats weighing approximately 250-300 $\mathrm{g}$ were purchased from the 



Figure 1 The operation procedure of VCA models. (A) The donor sites of BN rat, (B) the receptive site of SD rat, (C) the vascularized epigastric flap was harvested by cutting off the femoral artery/vein at the lower edge of the inguinal ligament, (D) the harvested allograft from $\mathrm{BN}$ rat, (E) the vascular anastomosis under operating microscope, (F) the established VCA model. VCA, vascularized composite allotransplantation; BN, Brown Norway; SD, Sprague Dawley.

Laboratory Animal Department of Central South University. The testing animals were kept in specific pathogen-free conditions. The animal studies were approved by the ethics committee of Xiangya Hospital of Central South University (No. 2020sydw0433) before implementation, in compliance with the national guidelines for the care and use of animals.

A BN allograft was transplanted to a SD recipient as described by Casal (34) and Ding (35). Under anesthesia by intraperitoneal injection of $3 \%(\mathrm{w} / \mathrm{v})$ pentobarbitone, the vascularized epigastric flap was harvested by cutting off the femoral artery/vein at the lower edge of the inguinal ligament from the $\mathrm{BN}$ donor. The femoral artery/vein of the flap was anastomosed with the carotid artery and external jugular vein of the SD recipient at the level of a recently created carotid opening, using 11/0 nylon sutures and the flap was secured at the site where the skin had been removed. The wound was closed with interrupted sutures using $4 / 0$ nylon sutures. The operation procedure is shown in Figure 1.

\section{Grouping of animals}

The allograft flap transplantation model was established using male $\mathrm{BN}$ rats as donors and $\mathrm{SD}$ rats as recipients.
Sixty-five SD rats underwent transplant, of which 17 were excluded from the study because flap failure within postoperative 3 days. Forty-eight SD rats were randomly by random number table divided into six equal groups ( $\mathrm{n}=8$ in each): no treatment (group I, control); empty PLGA nanoparticles (group II, NPs); single FK506 intraperitoneal (IP) injection (group III, S-FK506); single FK506 NPs IP injection (group IV, S-FK NPs); FK506 IP injection every 25 days (group V, M-FK506); FK506 NPs IP injection every 60 days (group VI, M-FK NPs). The first dose of immunosuppression in treatment groups was given at once after surgery (POD 0). Every administration contained $6 \mathrm{mg}$ of FK506, and up to 4 doses were administered to multiple injection groups (group V, M-FK506 and group VI, M-FK NPs). The injection intervals in the M-FK506 group and the M-FK NPs group were based on the pilot trial in FK506 blood concentration.

\section{Observation indicators}

\section{Graft survival time}

The flap conditions, such as rejection and hair growth, of each group were observed daily postoperatively, and graft 
rejection was evaluated macroscopically and graded as 0 (no rejection), 1 (erythema and edema), 2 (epidermolysis and exudation), and 3 (desquamation, necrosis, and mummification) (27). The endpoints were 60-day and 200-day allograft survival in single and multiple administration groups, respectively, or grade 3 rejection.

\section{FK506 blood concentration}

Ethylene diamine tetraacetic acid (EDTA) plasma samples were collected every 2 days after administration of FK506 in M-FK506 group and stored at $-80{ }^{\circ} \mathrm{C}$ until analysis. In M-FK NPs group, the samples were collected every 2 days in the first 10 days and then every 5 days until the next administration. Fifty microliters of EDTA plasma were analyzed using a PRO-Trac II ${ }^{\mathrm{TM}}$ Tacrolimus ELISA kit (DiaSorin Inc., Stillwater, MN, USA) according to the manufacturer's instructions.

\section{Flow cytometry analysis of $T$ regulatory cells (Tregs)}

Peripheral blood mononuclear cells (PBMCs) were collected by Ficoll density gradient centrifugation on postoperative days (PODs) 3, 9, and 12 in control and NPs groups; 3, 7, 14, and 25 in S-FK506 group; and 3, 9, 30, and 65 in S-FK NPs group. Briefly, PBMCs were stained with anti-rat monoclonal antibodies (mAbs) anti-CD4-PE and anti-CD25-APC (eBioscience Inc., San Diego, California, USA) for $30 \mathrm{~min}$, and then incubated with anti-FoxP3 mAbs (eBioscience) for $50 \mathrm{~min}$ after fixation and permeabilized by the Foxp3/Transcription Factor Staining Buffer Set (eBioscience). After staining, PBMCs were analyzed by flow cytometry using a SORP LSRII flow cytometer and BD Diva Software (BD Biosciences, San Jose, CA, USA). Data were analyzed using FlowJo ${ }^{\mathrm{TM}}$ software Version 10 (Tree Star Inc., Ashland, OR, USA).

\section{Plasma cytokine levels}

Levels of interleukins (IL)-2, IL-6, interferon (IFN)- $\gamma$, and transforming growth factor (TGF) $\beta$ in the plasma were evaluated at PODs 3, 7, 10, 20, 25, 30, 50, and 60 in control, S-FK506, and S-FK NPs groups. Briefly, $0.5-1.0 \mathrm{~mL}$ of tail vein blood was collected from each subject, and centrifuged at 3,000 rpm for $5 \mathrm{~min}$ to collect the supernatant, which was then frozen at $-20{ }^{\circ} \mathrm{C}$. Ten microliters of supernatant were used to detect the changes in the concentrations of these cytokines using the ELISA kits (eBioscience).

\section{Graft pathological examination}

The rats were euthanized by intraperitoneal injection of an overdose of pentobarbital. The graft tissue samples were collected on PODs 7 and 10 in control group and PODs 7, 15, and 30 in S-FK NPs group. The samples were preserved in $4 \%$ buffered formaldehyde and stained with hematoxylin and eosin, whereas paraffin sections were used for immunohistochemical (IHC) staining. The histological grading of skin rejection was performed according to the Banff classification (36).

Briefly, after a series of deparaffinization, rehydration, antigen retrieval, and blocking, sections were incubated with rabbit anti-mouse CD4 antibody (Abcam, Cambridge, UK) at $37^{\circ} \mathrm{C}$ for $60 \mathrm{~min}$. Primary antibodies were then detected by incubation with goat anti-rat IgG polymer (PV-9004, ZSGB-BIO, Beijing), followed by DAB staining (ZLI-9017, ZSGB-BIO, Beijing). The IHC staining results were scored by positive cell rate (PR) and staining intensity. PR Score $0:<10 \% ; 1: 10-25 \% ; 2: 25-50 \% ; 3: 50-75 \% ; 4:>75 \%$. Staining Score 1: weak staining; 2 : moderate staining; and 3: strong staining. $\mathrm{CD}^{+} \mathrm{T}$ cell infiltration $\mathrm{IHC}$ score $=\mathrm{PR} \times$ staining. IHC score $<1,(-) ; 1.1-2.0(+) ; 2.1-3.0(++) ; 3.1-4.0$ $(+++) ; \geq 4.0(++++)(37,38)$.

\section{Examination of liver and kidney functions}

Creatinine, blood urea nitrogen, aspartate aminotransferase, and alanine aminotransferase levels were measured as indicators of side effects due to the use of tacrolimus. Plasma samples were taken on POD 100 from the nonoperation group, M-FK506 and M-FK NPs group, and on POD 200 from M-FK NPs group. Creatinine, urea nitrogen, aspartate aminotransferase, and alanine aminotransferase (Jining Shiye, Shanghai) were assayed using a Hitachi 7020 automatic analyzer (Hitachi Ltd., Japan) according to the standard protocol in the enclosed pamphlets.

\section{Statistical analysis}

SPSS Version 21.0 (IBM Corp., Armonk, NY, USA) and GraphPad Prism Version 5.0 (GraphPad Software, San Diego, CA, USA) were used for statistical analysis and plotting graphs, respectively. The measurement data and results were reported as mean \pm standard deviation. The differences in survival time among various groups were compared and validated by the survival analysis log-rank test. Pairwise comparisons among multiple independent samples were performed using the one-way ANOVA-LSD test. The counting data were validated by chi-square $\left(\chi^{2}\right)$ test. $\mathrm{P}<0.05$ was considered as statistically significant. 


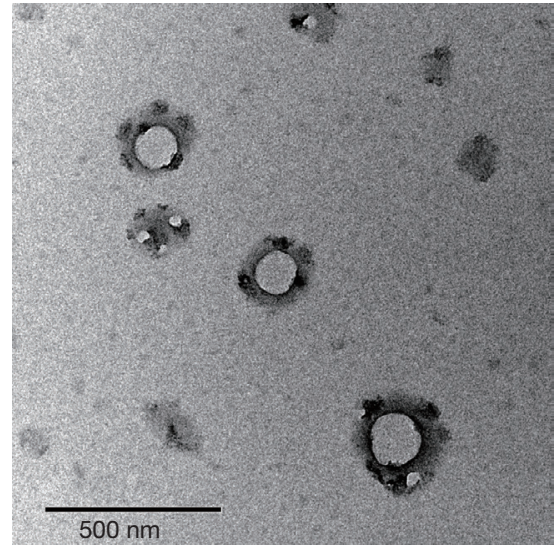

Figure 2 The establishment of FK506-loaded PLGA nanoparticles. The transmission electron microscope image of FK506 NPs (magnification, $20 \mathrm{kx}$, the scale bar is $500 \mathrm{~nm}$ ) and the average particle size of PLGA nanoparticles is $220 \pm 10 \mathrm{~nm}$. PLGA, poly lactic-co-glycolic acid; NPs, nanoparticles.

\section{Results}

\section{Preparation and characterization of FK506 NPs}

Under the electron microscope, both FK506 NPs and NPs were similar in morphology and size, both having regular, smooth spheroidal cores, with an average particle size of $220 \pm 10 \mathrm{~nm}$ (Figure 2). The encapsulation efficiency and drug loading of FK506 NPs were found to be $85.73 \% \pm 1.73 \%$ and $7.42 \% \pm 0.42 \%$.

\section{FK506 NPs promote long-term survival in a rat VCA transplantation model}

After transplantation, the mean survival times (MSTs) of allografts were $12.0 \pm 1.3,11.6 \pm 1.1,26.0 \pm 2.1$, and more than 60 days in control, NPs, S-FK506 and S-FK NPs group, respectively $(\mathrm{P}=0.54$, group control vs. $\mathrm{NP} ; \mathrm{P}<0.05$, group M-FK NPs $v s$. control, NPs and S-FK506; and $\mathrm{P}<0.05$, group S-FK506 vs. control and NPs). The MSTs of groups M-FK506 and M-FK NPs were $112.4 \pm 4.2$ days and more than 236 days $(\mathrm{P}<0.05$, group M-FK506 vs. M-FK NPs). The MSTs were significantly prolonged in both single and multiple administration groups of FK506 NPs (Figure 3 and Table 1).

\section{FK506 NPs maintain a longer effective blood drug concentration}

Plasma levels of FK506 were compared between groups
M-FK506 and M-FK NPs. Direct administration of $6 \mathrm{mg}$ of FK506 intraperitoneally after transplantation reached $207.2 \pm 17.6 \mathrm{ng} / \mathrm{mL}$ on POD 2 in group M-FK506. In group M-FK NPs, the FK506 concentration was notably lower $(29.3 \pm 4.0 \mathrm{ng} / \mathrm{mL})$ than in group M-FK506 $(\mathrm{P}<0.05)$. FK506 levels rapidly dropped to $103.1 \pm 13.5 \mathrm{ng} / \mathrm{mL}$ on day 6 , and was $5.4 \pm 1.2 \mathrm{ng} / \mathrm{mL}$ on day 25 in group M-FK506. In contrast, FK506 levels decreased much more slowly in group M-FK NPs. On POD 25, the concentration of FK506 in group M-FK NPs $(8.1 \pm 1.9 \mathrm{ng} / \mathrm{mL})$ was higher than that in group M-FK506 ( $\mathrm{P}=0.005)$, and then gradually decreased to $5.2 \pm 1.3 \mathrm{ng} / \mathrm{mL}$ on POD 60 . After re-administration, systemic FK506 levels demonstrated consistent circulating drug level curves (Figure 4).

\section{Proportion of Tregs during immunosuppression maintenance exceeds the inflammation period}

On POD 3, the proportion of Tregs was relatively low in all groups and was maintained at $2.2 \% \pm 0.1 \%$ and $2.3 \% \pm 0.1 \%$ in groups control and NPs, respectively $(\mathrm{P}=0.255$, group control vs. NPs). In Group S-FK506, The Treg proportion was $8.0 \% \pm 2.1 \%$ and $9.3 \% \pm 3.9 \%$ on POD 9 and 12 , and then dropped to $2.8 \% \pm 0.8 \%$ on POD 25 when rejection occurred ( $\mathrm{P}<0.05$, POD 9, 12 vs. 3, 25 in group S-FK506). As for group S-FK NPs, the average proportion of Tregs was $2.1 \% \pm 0.8 \%, 8.0 \% \pm 1.4 \%, 9.3 \% \pm 2.1 \%$, and $2.7 \% \pm 0.6 \%$ on PODs 3, 9, 30 and 65, respectively. The proportions of Tregs on POD 9 and 30 were significantly higher than on POD 3 and 65 in Group S-FK NPs $(\mathrm{P}<0.05$, POD 9, 30 vs. 3, 65 in Group S-FK NPs), and there were no statistical differences between groups S-FK506 and S-FK NPs during the allograft survival period $(\mathrm{P}>0.05, \mathrm{POD} 9,12$ in group S-FK506 vs. POD 9, 30 in group S-FK NPs; Figure 5 and Figure S1).

\section{FK506 NPs reduce inflammatory cytokines}

Inflammatory cytokines were induced in the rejection period and suppressed during immunosuppressive maintenance. The systemic levels of inflammatory cytokines in the control group were significantly higher than those in the FK506-treated groups on POD $10(\mathrm{P}<0.01)$. The levels of inflammatory cytokines in group S-FK NPs were significantly lower than those in group S-FK506 on POD 25 when rejection occurred in group S-FK506 but not in group S-FK NPs $(\mathrm{P}<0.01)$. The cytokine concentrations peaked on POD 60 when inflammation due to rejection was 
A
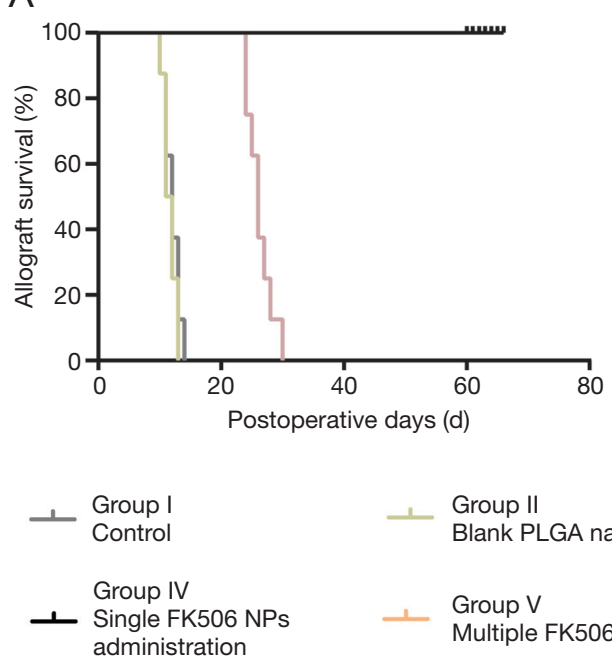

B

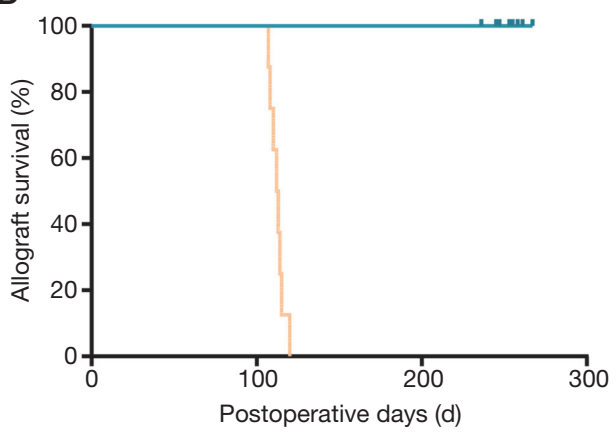

$+\quad$ Group III

Single FK506 administration

Group VI

1 Multiple FK506 NPs

administration

Figure 3 Survival time of each group. (A) The survival curve of single administration groups and (B) multiple administration groups. The mean survival times of FK506-loaded PLGA nanoparticles groups are significantly longer than other groups in both single and multiple administration groups. PLGA, poly lactic-co-glycolic acid.

Table 1 The mean survival time of each group.

\begin{tabular}{lllcc}
\hline Group & Treatment & $\mathrm{n}$ & MST (days) & P \\
\hline I & No treatment & 8 & $12.0 \pm 1.3$ & - \\
II & Blank PLGA nanoparticles & 8 & $11.6 \pm 1.1$ & II vs. I: P=0.54 \\
III & Single FK506 IP & 8 & $26.0 \pm 2.1$ & III vs. I, II: P<0.05 \\
IV & Single FK506 NPs IP & 8 & $>60$ & IV vs. I, II, III: P<0.05 \\
V & Multiple FK506 IP & 8 & $112.4 \pm 4.2$ & VI vs. V: P<0.05 \\
VI & Multiple FK506 NPs IP & 8 & $>236$ & \\
\hline
\end{tabular}

MST, mean survival time; PLGA, poly lactic-co-glycolic acid; IP, intraperitoneal injection; NPs, nanoparticles.

augmented in group S-FK NPs (Figure 6).

\section{FK506 NPs prolong immunosuppression}

All recipients in group control showed acute rejection of the graft with edema formation and necrosis. Disruption of the skin architecture was observed on POD 7 and 10, accompanied by mononuclear cells and $\mathrm{CD}^{+} \mathrm{T}$ helper cell infiltration. The histopathological changes were notably more severe on POD 10 than on POD 7 in the control group; the Banff and IHC scores on POD 7 and 10 were Banff 2, IHC (++) and Banff 3, IHC (+++), respectively. In group S-FK NPs, there was no destruction of the skin tissue structure, and mononuclear cell and $\mathrm{CD}^{+}{ }^{+} \mathrm{T}$ helper cell infiltration were less severe on POD 7 than the control group. On POD 15, mononuclear cells, and $\mathrm{CD}^{+}{ }^{+} \mathrm{T}$ helper cell infiltration vanished. Hair regrew on the skin graft on POD 30, no evident histopathological abnormalities were detected (Figure 7).

\section{FK506 NPs are less nephrotoxic}

The blood urea nitrogen and creatinine levels were notably higher in group M-FK506 $(20.9 \pm 1.5 \mathrm{mmol} / \mathrm{L}$ and $53.0 \pm 7.6 \mu \mathrm{mol} / \mathrm{L}$, respectively) than in the control and M-FK NPs groups (both POD 100 and 200) $(P<0.01)$. The urea nitrogen in group M-FK NPs $(10.5 \pm 1.0$ and $10.7 \pm 1.1 \mathrm{mmol} / \mathrm{L}$ on POD 100 and 200, respectively) was also higher than 

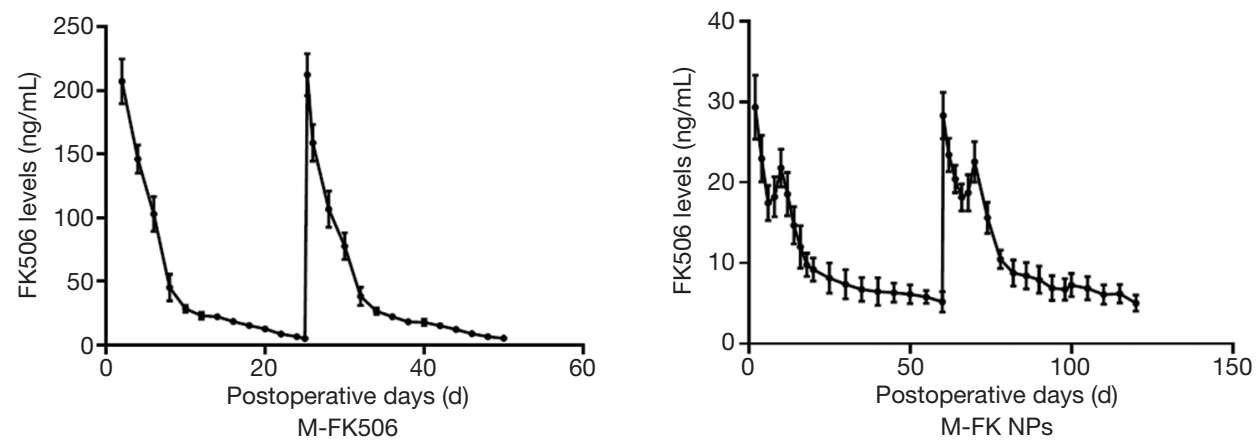

Figure 4 Systemic FK506 levels in group V (M-FK506) and group VI (M-FK NPs). (M-FK506) The initial burst release of tacrolimus IP peaks to $207.2 \pm 17.6 \mathrm{ng} / \mathrm{mL}$ on POD 2, then rapidly fell to $5.4 \pm 1.2 \mathrm{ng} / \mathrm{mL}$ on POD 25. (M-FK NPs) The concentration of FK506 decreases with time, and slightly increases during POD 6 to 10 , and then gradually drop to $5.2 \pm 1.3 \mathrm{ng} / \mathrm{mL}$ at POD 60 . The release pattern of FK506 in group M-FK NPs is much flatter than group M-FK506. IP, intraperitoneal injection; POD, postoperative days.

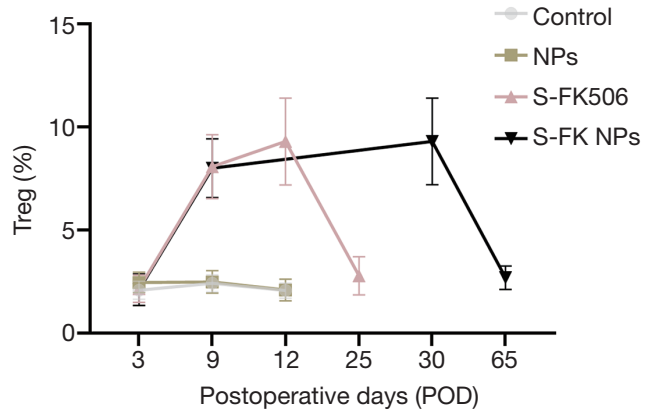

Figure 5 Treg subsets proportion in single administration groups. Flow cytometry of $\mathrm{CD}^{+} \mathrm{CD} 25^{+} \mathrm{FoxP}^{+}$Tregs in peripheral blood in single administration groups. The Treg proportions of group I and II fluctuate at low levels. The Treg proportion during immunosuppressive period (POD 9, 12 in group III and POD 9, 30 in group IV) are significantly higher than inflammatory rejection period ( $\mathrm{P}<0.05$, POD 9, 12 vs. 3, 25 in group III; POD 9, 30 vs. 3, 65 in group IV). CD, cluster of differentiation; FoxP3, forkhead box P3.

that in the control group $(5.9 \pm 1.1 \mathrm{mmol} / \mathrm{L})$, but there was no difference in creatinine concentration between group M-FK NPs $(22.9 \pm 2.2$ and $21.8 \pm 3.2 \mu \mathrm{mol} / \mathrm{L}$ on POD 100 and 200$)$ and the control group $(21.1 \pm 2.0 \mu \mathrm{mol} / \mathrm{L})(\mathrm{P}=0.11$, control group vs. group M-FK NPs). However, no statistically significant differences in aspartate aminotransferase and alanine aminotransferase concentrations were found among all groups (Figure 8).

\section{Discussion}

In clinical VCAs, long-term and regular administration of immunosuppressants is imperative to avoid graft loss due to acute or chronic rejection. New strategies are required to improve patient adherence whilst reducing the side effects of long-term use of immunosuppressants.

Our data indicate that injection of FK506 NPs after transplantation prolongs allograft survival. In our study, $6 \mathrm{mg}$ of tacrolimus encapsulated in PLGA as a one-time injection prevented allograft rejection for $>60$ days, which was also significantly longer than the MST of $26.0 \pm 2.1$ days in the $6 \mathrm{mg}$ tacrolimus intraperitoneal injection group. Sustained, targeted, and controlled release of drugs contributes to allograft survival (39). Unlike the systemic administration of FK506, FK506 NPs exhibited a biphasic release pattern, characterized by an initial burst release followed by a slower release $(31,40,41)$. The injection which contained tacrolimus to prevent FK506 NPs degradation before administration and the initial burst release of FK506 NPs caused the high systemic level in the immediate posttransplantation period. With the elimination of tacrolimus contained in the injection, the concentration gradient and the hydrolysis of the PLGA polymer accelerated the tacrolimus release in the POD 6-10 (40). In the following phase, tacrolimus is released progressively through the drug depleted layer. The therapeutic blood concentration of FK506 $(5-15 \mathrm{ng} / \mathrm{mL})(7,8,42)$ maintenance time of FK506 NPs (60 days) was notably longer than that of FK506 administration $(25$ days $)(\mathrm{P}<0.05)$, which suggests that the extended release of tacrolimus at therapeutic systemic levels can prevent immune activation. This is in line with the sustained tacrolimus levels contributing to the inhibition of immune cell function by daily topical application of tacrolimus (40). The extended release of tacrolimus also 

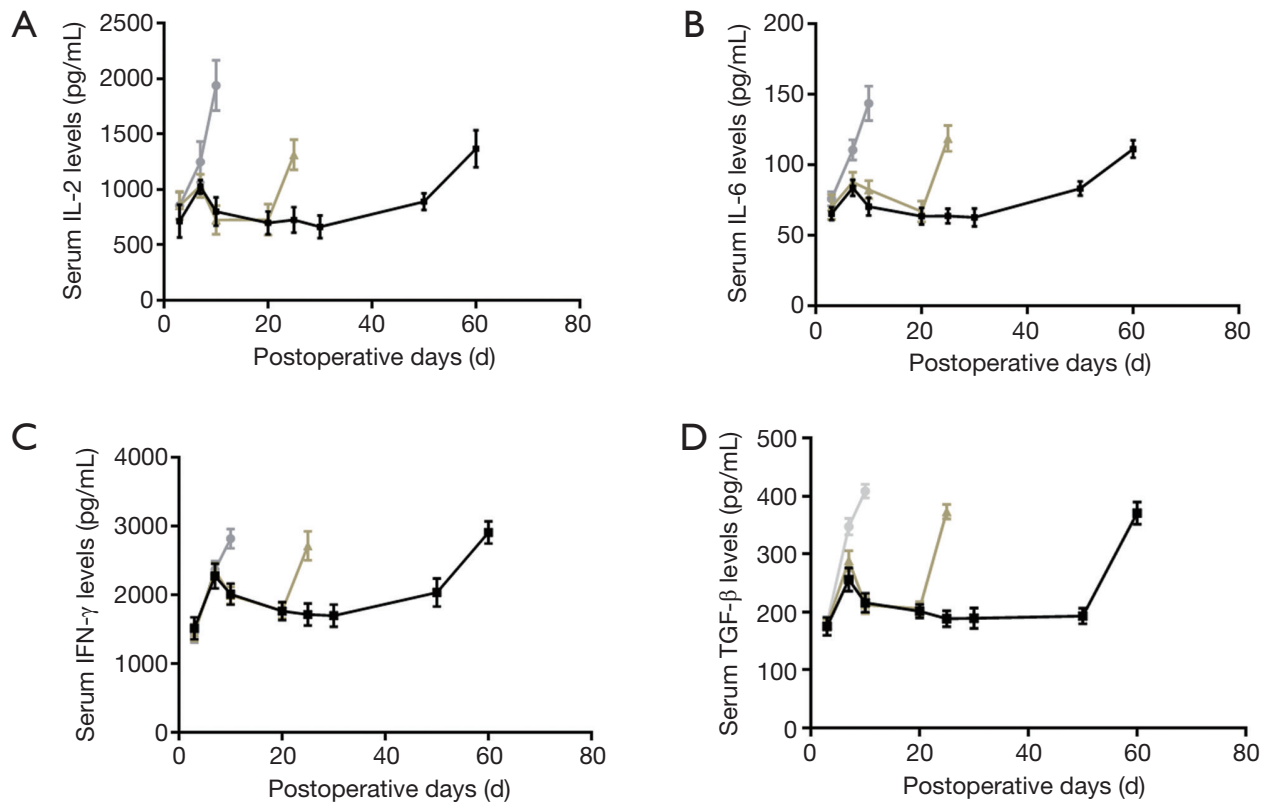

$\rightarrow$ Group I $\quad$ - Group III $\quad$ - Group IV

Figure 6 Systemic levels of pro-inflammatory cytokines in groups I, III and IV. The concentrations of (A) IL-2, (B) IL-6, (C) IFN- $\gamma$ and (D) TGF- $\beta$ in group I increase with time, and those concentration were significantly higher than FK506 treated groups III and IV on POD 10 $(\mathrm{P}<0.01$, group I vs. III; IV). The concentrations of cytokines rapidly increase after POD 20 in group III, and significantly higher than group IV on POD $25(\mathrm{P}<0.01)$. The gradual increases of pro-inflammatory cytokines occur after POD 30, and peaked on POD 60 in group IV. IL, interleukin; IFN- $\gamma$, interferon $\gamma$; TGF- $\beta$, transforming growth factor $\beta$; POD, postoperative days.

means longer administration intervals which may improve medication adherence $(43,44)$. In addition, the steadystate systemic levels of tacrolimus achieved by FK506 NPs prevents high systemic tacrolimus levels (as seen in tacrolimus IP injection), which may lead to toxicity (45-48). The steady-state systemic level of tacrolimus is also clinically significant for reducing the side effects of highly variable systemic tacrolimus levels in daily parenteral administration (29,49-51).

In our study, as in others, Treg upregulation was closely related to the prolongation of allograft survival (52,53). Lin et al. (54) achieved conventional $\mathrm{CD}^{+}{ }^{+}$Tregs in the peripheral blood of tolerant VCA recipients in a mouse vascularized osteomyocutaneous transplantation model. In another model of heterotopic hind limb VCA in rats in which repetitive use of syngeneic adiposederived mesenchymal stem cells promoted long-term allograft survival, there was sustained circulating Tregs levels observed (55). To some extent, the increase in the proportion of Treg represents the induction of immune tolerance $(56,57)$. As for the inflammatory cytokines, different cytokines have potential as markers for rejection $(58,59)$. We observed increased levels of several cytokines (IL-2, IL-6, IFN- $\gamma$, and TGF- $\beta$ ) in the first week after operation and after the diminishment of tacrolimus, which is consistent with a previous study (27). Liu et al. (60) showed that FK506 loaded nanomicelles can remarkably reduce the amount of IL-2 and IL-17 to sustain the immunosuppressive effect. In a dynamic observation of serum cytokines in patients with hand transplantation for 5 months, it has been demonstrated that the low level of inflammatory cytokines (IL-2, IFN- $\gamma$, and TNF- $\alpha$ ) is concordant with the survival of transplanted hands (61). As a result, FK506-loaded nanoparticles exhibited a strong immunosuppressive effect in the prevention of allograft rejection by sustaining higher Tregs proportion and prohibiting inflammatory cytokines.

Our data show that repeated intraperitoneal injection of tacrolimus-loaded PLGA nanoparticles sustained longterm graft survival with better toxicological outcomes than intraperitoneal injection of tacrolimus. Acute kidney injury was observed in group M-FK but not in group M-FK NPs. 



Figure 7 Histopathological features of vascular composite allograft in the control group (A,B) and group S-FK NPs (C-E). Representative macroscopic and photomicrographs of the histology (hematoxylin and eosin staining; CD4 immunohistochemical staining) images of allografts at POD 7 (A) and POD 10 (B) in the control group; and POD 7 (C), POD 15 (D) and POD 30 (E) in group S-FK NPs. Scale bar $=400 \mu \mathrm{m}$. (A) $\mathrm{A}_{1}$ showed an acute rejection at 7 days, $\mathrm{A}_{2}$ showed a moderate amount of mononuclear cell infiltration in the dermis layer, the epidermis was intact. $A_{3}$ showed lightly staining in the lymphoid follicles and inter-lymphoid follicles. (B) $B_{1}$ showed deteriorated rejection on POD 10, the infiltration of mononuclear cell in the dermis layer increase, the epidermal cells were swollen $\left(\mathrm{B}_{2}\right)$, the staining in the lymphoid follicles and inter-lymphoid follicles deepened $\left(\mathrm{B}_{3}\right)$. The Banff and IHC scores on POD 7 and 10 were Banff 2, IHC (++) and Banff 3, IHC (+++), respectively. No signs of rejection were seen in group IV at day $7\left(\mathrm{C}_{1}\right), 15\left(\mathrm{D}_{1}\right)$ and $30\left(\mathrm{E}_{1}\right)$. The skin structure was normal on POD 7 in group IV, and a small amount of lymphocyte infiltration were observed, the Banff' classification is grade $1\left(\mathrm{C}_{2}\right)$, and there is weakly positively staining in the lymphoid follicles and inter-lymphoid follicles $\left(\mathrm{C}_{3}\right)$. On POD 15 and 30 in group $\mathrm{IV}$, no abnormalities detected macroscopically and microscopically $\left(\mathrm{D}_{2}, \mathrm{D}_{3} ; \mathrm{E}_{2}, \mathrm{E}_{3}\right)$. POD, postoperative days; IHC, immunohistochemical. 



Figure 8 Indicator of kidney and liver functions. (A) Blood urea nitrogen level in group M-FK506 is significantly higher than the control group and group M-FK NPs on POD 100 and 200, and the concentrations of urea nitrogen in group M-FK NPs on POD 100 and 200 are also higher than the control group. (B) The amount of creatinine in group M-FK506 is significantly higher than the control group and group M-FK NPs on POD 100 and 200, while there is no statistical difference between the control group and group M-FK NPs. There is no statistical difference of aspartate aminotransferase and alanine aminotransferase concentrations among all groups (C,D) (ns, $\mathrm{P}>0.05$; $\left.{ }^{*}, \mathrm{P}<0.05\right)$. POD, postoperative days.

Acute nephrotoxicity has been attributed to systemic and renal vasoconstriction, leading to reduced renal blood flow and glomerular filtration rate, which is dose-related and reversible (62). The smaller fluctuations and lower systemic tacrolimus levels obtained by sustained release of FK506 NPs are beneficial to preserving kidney function.

As the one of the most commonly used biodegradable copolymer, PLGA was initially used as suture materials in humans $(63,64)$. Due to its excellent biodegradability, biosafety and biocompatibility, PLGA has been approved by U.S. food and Drug Administration (FDA) as drug delivery carrier, tissue engineering scaffolds and resorbable prosthetic devices $(65,66)$. Since the first approval of Lupron Depot based on PLGA microparticles in January 1989 by the U.S. FDA, only 20 drug products of injection delivery based on PLGA formulations have been presented on the pharmaceutical market, of which 13 are micro-/ nanoparticles, 3 are solid implant and 4 are in situ gelforming implant $(67,68)$. These clinical application of PLGA formulations have contributed to treatment of numerous diseases including cancer, diabetes or different immune disorders. On the other hand, the complicated design of a safe and effective PLGA formulation and the acidic micro-environment resulted from the hydrolysis of PLGA limit the clinical application $(69,70)$. In addition, a more effective "on-demand" release of encapsulated molecules should be developed to enhance therapeutic efficacy. The negligible number approved by the U.S. FDA indicates the study and use of clinical PLGA formulations will continues to be a challenging field. 
One of the major limitations of this study is intra-graft levels of tacrolimus were not tested. Several studies have highlighted the lack of a relationship between intracellular and blood concentration for tacrolimus (71), and the intra-graft level of immunosuppressive drugs is a more accurate marker of immunosuppression than trough levels. Therefore, pharmacokinetic studies analyzing tacrolimus intra-graft and intracellular distribution are needed. Additionally, our study is a rodent study only; further research on large animals such as pigs is required to evaluate the feasibility of FK506 NPs. IP injection is not likely to be applied in VCA recipients, indicating that the effect of different administration routes such as intragraft injection or intravenous injection on the systemic and intragraft distribution of FK506 needs to be investigated.

\section{Conclusions}

Drug administration through FK506 NPs prolonged the survival time of flap allografts with less nephrotoxicity in a rodent model. However, further research in large animals, such as pigs, is needed before transition to humans. The effects of long-term application of PLGA nanoparticles also need to be investigated.

\section{Acknowledgments}

Funding: This work was supported by the National Natural Science Foundation of China [Grant No.817081927]; the National Natural Science Foundation of China [Grant No. 81871577]; the National Natural Science Foundation of China [Grant No. 81901978]; the Natural Science Foundation of Hunan Province, China [Grant No. 2018JJ6056].

\section{Footnote}

Reporting Checklist: The authors have completed the ARRIVE reporting checklist. Available at https://dx.doi. org/10.21037/atm-21-2425

Data Sharing Statement: Available at https://dx.doi. org/10.21037/atm-21-2425

Conflicts of Interest: All authors have completed the ICMJE uniform disclosure form (available at https://dx.doi. org/10.21037/atm-21-2425). The authors have no conflicts of interest to declare.
Ethical Statement: The authors are accountable for all aspects of the work in ensuring that questions related to the accuracy or integrity of any part of the work are appropriately investigated and resolved. Animal experiments were approved by the ethics committee of Xiangya Hospital of Central South University (No. 2020sydw0433), in compliance with the national guidelines for the care and use of animals.

Open Access Statement: This is an Open Access article distributed in accordance with the Creative Commons Attribution-NonCommercial-NoDerivs 4.0 International License (CC BY-NC-ND 4.0), which permits the noncommercial replication and distribution of the article with the strict proviso that no changes or edits are made and the original work is properly cited (including links to both the formal publication through the relevant DOI and the license). See: https://creativecommons.org/licenses/by-nc-nd/4.0/.

\section{References}

1. Hofmann GO, Kirschner MH, Brauns L, et al. Vascularized knee joint transplantation in man: a report on the first cases. Transpl Int 1998;11 Suppl 1:S487-90.

2. Dubernard JM, Owen E, Herzberg G, et al. Human hand allograft: report on first 6 months. Lancet 1999;353:1315-20.

3. Dubernard JM, Petruzzo P, Lanzetta M, et al. Functional results of the first human double-hand transplantation. Ann Surg 2003;238:128-36.

4. Devauchelle B, Badet L, Lengelé B, et al. First human face allograft: early report. Lancet 2006;368:203-9.

5. Breidenbach WC, Gonzales NR, Kaufman CL, et al. Outcomes of the first 2 American hand transplants at 8 and 6 years posttransplant. J Hand Surg Am 2008;33:1039-47.

6. Grajek M, Maciejewski A, Giebel S, et al. First Complex Allotransplantation of Neck Organs: Larynx, Trachea, Pharynx, Esophagus, Thyroid, Parathyroid Glands, and Anterior Cervical Wall: A Case Report. Ann Surg 2017;266:e19-24.

7. Petruzzo P, Lanzetta M, Dubernard JM, et al. The International Registry on Hand and Composite Tissue Transplantation. Transplantation 2010;90:1590-4.

8. Petruzzo P, Sardu C, Lanzetta M, et al. Report (2017) of the International Registry on Hand and Composite Tissue Allotransplantation (IRHCTT). Curr Transplant Rep 2017;4:294-303.

9. Morelon E, Petruzzo P, Kanitakis J. Chronic rejection in 
vascularized composite allotransplantation. Curr Opin Organ Transplant 2018;23:582-91.

10. Kino T, Hatanaka H, Miyata S, et al. FK-506, a novel immunosuppressant isolated from a Streptomyces. II. Immunosuppressive effect of FK-506 in vitro. J Antibiot (Tokyo) 1987;40:1256-65

11. Morris R. Modes of action of FK506, cyclosporin A, and rapamycin. Transplant Proc 1994;26:3272-5.

12. Bonastre J, Landin L, Diez J, et al. Factors influencing acute rejection of human hand allografts: a systematic review. Ann Plast Surg 2012;68:624-9.

13. Brandacher G, Lee WP, Schneeberger S. Minimizing immunosuppression in hand transplantation. Expert Rev Clin Immunol 2012;8:673-83; quiz 684.

14. Miller LW. Cardiovascular toxicities of immunosuppressive agents. Am J Transplant 2002;2:807-18.

15. Tanabe K. Calcineurin inhibitors in renal transplantation: what is the best option? Drugs 2003;63:1535-48.

16. Staatz CE, Tett SE. Clinical pharmacokinetics and pharmacodynamics of tacrolimus in solid organ transplantation. Clin Pharmacokinet 2004;43:623-53.

17. Campagne O, Mager DE, Brazeau D, et al. The impact of tacrolimus exposure on extrarenal adverse effects in adult renal transplant recipients. Br J Clin Pharmacol 2019;85:516-29.

18. Cendales L, Bray R, Gebel H, et al. Tacrolimus to Belatacept Conversion Following Hand Transplantation: A Case Report. Am J Transplant 2015;15:2250-5.

19. K dzierska K, Sindrewicz K, Sporniak-Tutak K, et al. Does Immunosuppressive Therapy Affect Markers of Kidney Damage? Ann Transplant 2016;21:137-44.

20. Lamprecht A, Yamamoto H, Takeuchi H, et al. Design of $\mathrm{pH}$-sensitive microspheres for the colonic delivery of the immunosuppressive drug tacrolimus. Eur J Pharm Biopharm 2004;58:37-43.

21. Miyamoto Y, Uno T, Yamamoto H, et al. Pharmacokinetic and immunosuppressive effects of tacrolimus-loaded biodegradable microspheres. Liver Transpl 2004;10:392-6.

22. Shirali AC, Look M, Du W, et al. Nanoparticle delivery of mycophenolic acid upregulates PD-L1 on dendritic cells to prolong murine allograft survival. Am J Transplant 2011;11:2582-92.

23. Pham TT, Nguyen TT, Pathak S, et al. Tissue adhesive FK506-loaded polymeric nanoparticles for multilayered nano-shielding of pancreatic islets to enhance xenograft survival in a diabetic mouse model. Biomaterials 2018;154:182-96.

24. Deng C, Chen Y, Zhang L, et al. Delivery of FK506- loaded PLGA nanoparticles prolongs cardiac allograft survival. Int J Pharm 2020;575:118951.

25. Shi W, Liu T, Xie L, et al. FK506 in a biodegradable glycolide-co-clatide-co-caprolactone polymer for prolongation of corneal allograft survival. Curr Eye Res 2005;30:969-76.

26. Xu W, Ling $\mathrm{P}$, Zhang T. Toward immunosuppressive effects on liver transplantation in rat model: tacrolimus loaded poly(ethylene glycol)-poly(D,L-lactide) nanoparticle with longer survival time. Int J Pharm 2014;460:173-80.

27. Gajanayake T, Olariu R, Leclère FM, et al. A single localized dose of enzyme-responsive hydrogel improves long-term survival of a vascularized composite allograft. Sci Transl Med 2014;6:249ra110.

28. Wang S, Xiong Y, Wang Y, et al. Evaluation of PLGA microspheres with triple regimen on long-term survival of vascularized composite allograft - an experimental study. Transpl Int 2020;33:450-61.

29. O'Regan JA, Canney M, Connaughton DM, et al. Tacrolimus trough-level variability predicts long-term allograft survival following kidney transplantation. J Nephrol 2016;29:269-76.

30. Julienne MC, Alonso MJ, GÓMez Amoza JL, et al. Preparation of Poly(D,L-Lactide/Glycolide) Nanoparticles of Controlled Particle Size Distribution: Application of Experimental Designs. Drug Dev Ind Pharm 1992;18:1063-77.

31. Sánchez A, Vila-Jato JoséL, Alonso MJ. Development of biodegradable microspheres and nanospheres for the controlled release of cyclosporin A. Int J Pharm 1993;99:263-73.

32. Vallelado AI, López MI, Calonge M, et al. Efficacy and safety of microspheres of cyclosporin A, a new systemic formulation, to prevent corneal graft rejection in rats. Curr Eye Res 2002;24:39-45.

33. Kimura H, Ogura Y. Biodegradable polymers for ocular drug delivery. Ophthalmologica 2001;215:143-55.

34. Casal D, Pais D, Iria I, et al. A Model of Free Tissue Transfer: The Rat Epigastric Free Flap. J Vis Exp 2017;(119):55281.

35. Ding J, Su Y, Liu S, et al. A Mouse Model of Vascularized Skin Transplantation. Ann Plast Surg 2017;78:576-81.

36. Cendales LC, Kanitakis J, Schneeberger S, et al. The Banff 2007 working classification of skin-containing composite tissue allograft pathology. Am J Transplant 2008;8:1396-400.

37. McDonald JW, Pilgram TK. Nuclear expression of $\mathrm{p} 53$, 
p21 and cyclin D1 is increased in bronchioloalveolar carcinoma. Histopathology 1999;34:439-46.

38. Charafe-Jauffret E, Tarpin C, Bardou VJ, et al. Immunophenotypic analysis of inflammatory breast cancers: identification of an 'inflammatory signature'. J Pathol 2004;202:265-73.

39. Hubbell JA, Thomas SN, Swartz MA. Materials engineering for immunomodulation. Nature 2009;462:449-60.

40. Makadia HK, Siegel SJ. Poly Lactic-co-Glycolic Acid (PLGA) as Biodegradable Controlled Drug Delivery Carrier. Polymers (Basel) 2011;3:1377-97.

41. Sgorla D, Bunhak ÉJ, Cavalcanti OA, et al. Exploitation of lipid-polymeric matrices at nanoscale for drug delivery applications. Expert Opin Drug Deliv 2016;13:1301-9.

42. Chang J, Davis CL, Mathes DW. The impact of current immunosuppression strategies in renal transplantation on the field of reconstructive transplantation. J Reconstr Microsurg 2012;28:7-19.

43. Weng FL, Israni AK, Joffe MM, et al. Race and electronically measured adherence to immunosuppressive medications after deceased donor renal transplantation. J Am Soc Nephrol 2005;16:1839-48.

44. Lehner LJ, Reinke P, Hörstrup JH, et al. Evaluation of adherence and tolerability of prolonged-release tacrolimus (Advagraf ${ }^{\mathrm{TM}}$ ) in kidney transplant patients in Germany: A multicenter, noninterventional study. Clin Transplant 2018. doi: 10.1111/ctr.13142.

45. Przepiorka D, Nash RA, Wingard JR, et al. Relationship of tacrolimus whole blood levels to efficacy and safety outcomes after unrelated donor marrow transplantation. Biol Blood Marrow Transplant 1999;5:94-7.

46. Venkataramanan R, Shaw LM, Sarkozi L, et al. Clinical utility of monitoring tacrolimus blood concentrations in liver transplant patients. J Clin Pharmacol 2001;41:542-51.

47. Sikma MA, van Maarseveen EM, van de Graaf EA, et al. Pharmacokinetics and Toxicity of Tacrolimus Early After Heart and Lung Transplantation. Am J Transplant 2015;15:2301-13.

48. Sikma MA, Hunault CC, van de Graaf EA, et al.

High tacrolimus blood concentrations early after lung transplantation and the risk of kidney injury. Eur J Clin Pharmacol 2017;73:573-80.

49. Gueta I, Markovits N, Yarden-Bilavsky H, et al. High tacrolimus trough level variability is associated with rejections after heart transplant. Am J Transplant 2018;18:2571-8.

50. Pollock-Barziv SM, Finkelstein Y, Manlhiot C, et al.
Variability in tacrolimus blood levels increases the risk of late rejection and graft loss after solid organ transplantation in older children. Pediatr Transplant 2010;14:968-75.

51. Sapir-Pichhadze R, Wang Y, Famure O, et al. Timedependent variability in tacrolimus trough blood levels is a risk factor for late kidney transplant failure. Kidney Int 2014;85:1404-11.

52. Pilat N, Baranyi U, Klaus C, et al. Treg-therapy allows mixed chimerism and transplantation tolerance without cytoreductive conditioning. Am J Transplant 2010;10:751-62.

53. Issa F, Hester J, Goto R, et al. Ex vivo-expanded human regulatory $\mathrm{T}$ cells prevent the rejection of skin allografts in a humanized mouse model. Transplantation 2010;90:1321-7.

54. Lin CH, Zhang W, Ng TW, et al. Vascularized osteomyocutaneous allografts are permissive to tolerance by induction-based immunomodulatory therapy. Am J Transplant 2013;13:2161-8.

55. Cheng HY, Ghetu N, Huang WC, et al. Syngeneic adipose-derived stem cells with short-term immunosuppression induce vascularized composite allotransplantation tolerance in rats. Cytotherapy 2014;16:369-80.

56. Waldmann H, Adams E, Fairchild P, et al. Infectious tolerance and the long-term acceptance of transplanted tissue. Immunol Rev 2006;212:301-13.

57. Sakaguchi S, Yamaguchi T, Nomura T, et al. Regulatory T cells and immune tolerance. Cell 2008;133:775-87.

58. Borges TJ, O'Malley JT, Wo L, et al. Codominant Role of Interferon- $\gamma$ - and Interleukin-17-Producing T Cells During Rejection in Full Facial Transplant Recipients. Am J Transplant 2016;16:2158-71.

59. Wolfram D, Morandi EM, Eberhart N, et al. Differentiation between acute skin rejection in allotransplantation and T-cell mediated skin inflammation based on gene expression analysis. Biomed Res Int 2015;2015:259160.

60. Liu D, Wu Q, Chen W, et al. A novel FK506 loaded nanomicelles consisting of amino-terminated poly(ethylene glycol)-block-poly(D,L)-lactic acid and hydroxypropyl methylcellulose for ocular drug delivery. Int J Pharm 2019;562:1-10.

61. Zheng X, Pei G, Qiu Y, et al. Dynamic observation of serum cytokines in the patients with hand transplantation. Transplant Proc 2002;34:3405-9.

62. Peters DH, Fitton A, Plosker GL, et al. Tacrolimus. A 
review of its pharmacology, and therapeutic potential in hepatic and renal transplantation. Drugs 1993;46:746-94.

63. Conn J Jr, Oyasu R, Welsh M, et al. Vicryl (polyglactin 910) synthetic absorbable sutures. Am J Surg 1974;128:19-23.

64. Huh BK, Kim BH, Kim SN, et al. Surgical suture braided with a diclofenac-loaded strand of poly(lactic-co-glycolic acid) for local, sustained pain mitigation. Mater Sci Eng C Mater Biol Appl 2017;79:209-15.

65. Arakawa CK, DeForest CA. Chapter 19: Polymer Design and Development. In: Vishwakarma A, Karp JM. editors. Biology and Engineering of Stem Cell Niches. Boston: Academic Press, 2017:295-314.

66. Pandita D, Kumar S, Lather V. Hybrid poly(lacticco-glycolic acid) nanoparticles: design and delivery prospectives. Drug Discov Today 2015;20:95-104.

67. Park K, Skidmore S, Hadar J, et al. Injectable, longacting PLGA formulations: Analyzing PLGA and

Cite this article as: Cao Z, Li C, He J, Sui X, Wu P, Pan D, Qing L, Tang J. FK506-loaded PLGA nanoparticles improve long-term survival of a vascularized composite allograft in a murine model. Ann Transl Med 2021;9(20):1515. doi:10.21037/ atm-21-2425 understanding microparticle formation. J Control Release 2019;304:125-34.

68. Ghitman J, Biru EI, Stan R, et al. Review of hybrid PLGA nanoparticles: Future of smart drug delivery and theranostics medicine. Mater Des 2020;193:108805.

69. Estey T, Kang J, Schwendeman SP, et al. BSA degradation under acidic conditions: a model for protein instability during release from PLGA delivery systems. J Pharm Sci 2006;95:1626-39.

70. Ding D, Zhu Q. Recent advances of PLGA micro/ nanoparticles for the delivery of biomacromolecular therapeutics. Mater Sci Eng C Mater Biol Appl 2018;92:1041-60.

71. Capron A, Haufroid V, Wallemacq P. Intra-cellular immunosuppressive drugs monitoring: A step forward towards better therapeutic efficacy after organ transplantation? Pharmacol Res 2016;111:610-8. 


\section{Supplementary}


Figure S1 Flow cytometric cytograms of Tregs in the control group (A) and group S-FK NPs (B). Flow cytometric dot plot of CD4+ CD25+ FoxP3 + Tregs in peripheral blood in the control group on POD 3, 9, 12 (A1-A3) and in group S-FK NPs on POD 3, 9, 30 and 65 (B1-B4). The fluctuation of Treg proportions in group blank NPs is the same as in the control group; while the variations are the same in group S-FK506 and S-FK NPs. CD, cluster of differentiation; FoxP3, forkhead box P3; POD, postoperative days. 